\title{
Three-dimensional Structure of Eukaryotic Complex I
}

\author{
Todd A. Clason, ${ }^{*}$ Teresa Ruiz ${ }^{*}$, Hermann Schägger***, Michael Radermacher* \\ * Dept. Mol. Physiol. \& Biophysics, University of Vermont, Burlington, VT 05405, \\ ${ }^{* * *}$ Zentrum der Biologischen Chemie, Universitätsklinikum Frankfurt,D-60590 Frankfurt, Germany
}

Complex I (NADH dehydrogenase) is the first and largest enzyme of the mitochondrial respiratory chain. It couples the transfer of electrons from NADH to ubiquinone with the translocation of four protons across the membrane. Bacterial complex I has 14 subunits while eukaryotes have additional subunits, up to 46, and a molecular mass of about $1 \mathrm{MDa}$ in the bovine enzyme (for review see e.g. [1] ). The complete subunit architecture of complex I has not been determined yet and many aspects of its function are still highly speculative. Because of the difficulties in crystallizing membrane proteins and the large size of the molecule, it has not been possible to determine a high resolution $\mathrm{X}$-ray structure of this complex. Only recently the successful collection of X-ray data of the hydrophilic fragment of complex I has been reported [2].

Here we present the three-dimensional structure of bovine complex I purified by blue native gel electrophoresis. Bovine heart mitochondria were solubilized using dodecylmaltoside $(2.5 \mathrm{~g} / \mathrm{g}$ protein) and complex I was isolated by BN-PAGE as described in [3]. For microscopy purified complex I was embedded in stain (PTA pH 7.0) using a deep staining technique [4, 5]. The particles visible in the micrograph showed an excellent preservation of the enzyme.

For 3D reconstruction, tilt pairs were collected, with a tilt angle of approximately $55^{\circ}$. A total of 7730 particle images were extracted from 78 tilt pairs. All micrographs were corrected for the microscope transfer function according to [6] The untilted images were centered, aligned to an unbiased reference obtained by reference free alignment [7]. A first analysis with correspondence analysis and classification [8-10] split the data set approximately in half, according to the major orientation of the particles relative to the carbon support. These two classes were analyzed separately and subjected to multiple rounds of multi reference alignments and classification.

Two recent reconstructions of complex I $[12,13]$ are inconsistent with our new results, yet earlier reconstructions of the Escherichia coli and Neurospora crassa complex I [14, 15] can easily be reconciled. Our new structure shows a clear division of the matrix arm into distinct globular domains. When compared to our recent reconstruction of complex I from the aerobic yeast Yarrowia lipolytica [11], the domains visible in bovine complex I can be directly identified with the same domains in the yeast complex. These distinct protein domains now visible provide a solid framework for the identification of subunits and their three-dimensional arrangement.

[1] Brandt U., Ann. Rev. Biochem., 2006, 75, in press

[2] Hinchliffe P. and Sazanov, L. A., Science, 2005, 309, 5735, 771-4

[3] Schägger H., in: Membrane protein purification and crystallization, (C. Hunte, G. von Jagow and H. Schägger eds.), San Diego, 2003, Academic Press

[4] Stoops J. K., et al., Proc. Natl. Acad. Sci. USA, 1992, 89, 14, 6585-6589

[5] Ruiz T. and Radermacher, M., in: Methods in Molecular Biology: Cell imaging techniques: methods and protocols, (B. T. M. D. J. Taatjes eds.), Totowa, 2005, Humana Press Inc. 
[6] Radermacher M., et al., Journal of Structural Biology., 2001, 135, 1, 26-37

[7] Marco S., et al., Ultramicroscopy, 1996, 66, 5-10

[8] van Heel M. and Frank, J., Ultramicroscopy, 1981, 6, 2, 187-194

[9] Frank J. and van Heel, M., J. Mol. Biol., 1982, 161, 1, 134-137

[10] Diday E., Rev. Stat. Appl., 1971, 19, 19-34

[11] Radermacher M., et al., J. Struct. Biol., 2006, submitted

[12] Grigorieff N., J. Mol. Biol., 1998, 277, 5, 1033-46

[13] Böttcher B., et al., J. Biol. Chem., 2002, 277, 20, 17970-17977

[14] Guénebaut V., et al., J. Mol. Biol., 1997, 265, 409-418

[15] Guénebaut V., et al., J. Mol. Biol., 1998, 276, 1, 105-112

[16] Pettersen E. F., et al., J. Comput. Chem., 2004, 25, 1605-1612

[17] This work was supported by grant NIH RO1 1RO1 GM068650 (to M.R.) and DFG SFB472 (to H.S.) and has benefited from NIH RO1 GM069551 (to T.R.)

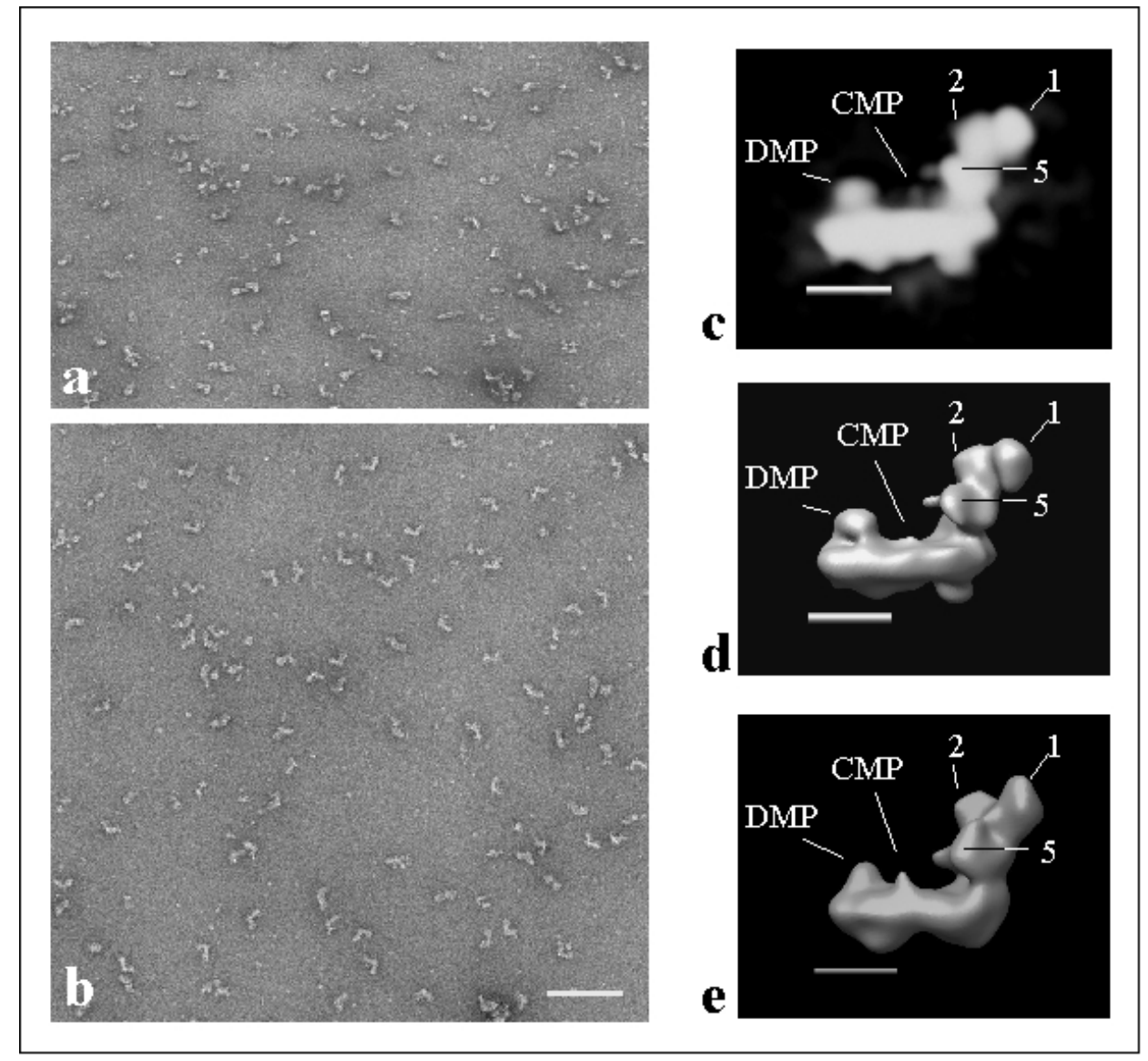

The reconstruction of bovine complex I. Left: Pair of micrographs a) specimen tilted by $\sim 55^{\circ}$, b) $0^{\circ}$ image of the same specimen area. Tilt axis horizontal. Scale bar $100 \mathrm{~nm}$. Right: c) and d) 3D reconstruction of bovine complex I. c) volume rendered, b) surface representation. Scale bar $10 \mathrm{~nm}$. c) Surface of the previous reconstruction of complex I from Yarrowia lipolytica. Scale bar $10 \mathrm{~nm}$. Some of the domains recognizable in the reconstructions from both species are indicated (domains 1, 2, and 5, the Central Membrane arm Protuberance (CMP) and the Distal Membrane arm Protuberance (DMP). Nomenclature used from [11].) All 3D representations were carried out with Chimera [16] 